\title{
Effectiveness of ultrasound-guided percutaneous transhepatic puncture for the diagnosis of low-level alpha-fetoprotein liver cancer patients
}

\author{
Yeliu Fu ${ }^{1 \#}$, Junyao Chen ${ }^{2 \#}$, Caiyang $\mathrm{Li}^{2}$, Lie Chen ${ }^{1}$, Zhilan Zhang ${ }^{1}$, Zhenxiu Huang ${ }^{3}$ \\ ${ }^{1}$ Department of Medical Ultrasonics, Central South University Xiangya School of Medicine Affiliated Haikou Hospital, Haikou, China; ${ }^{2}$ Department \\ of Ultrasonography, Hainan Cancer Hospital, Haikou, China; ${ }^{3}$ Department of Ultrasonography, Wanning People's Hospital, Wanning, China \\ Contributions: (I) Conception and design: Y Fu, J Chen, Z Huang; (II) Administrative support: C Li, L Chen, Z Zhang; (III) Provision of study \\ materials or patients: L Chen, Z Zhang; (IV) Collection and assembly of data: All authors; (V) Data analysis and interpretation: Y Fu, J Chen, Z \\ Huang; (VI) Manuscript writing: All authors; (VII) Final approval of manuscript: All authors. \\ \#These authors contributed equally to this work. \\ Correspondence to: Zhenxiu Huang. Department of Ultrasonography, Wanning People's Hospital, No. 1 Huanshisandong Road, Wanning 571500, \\ China. Email: huangzhengxiu1021@163.com.
}

Background: Globally, liver cancer is the most frequent fatal malignancy. The aim of the present study was to explore the effectiveness of ultrasound (US)-guided percutaneous transhepatic puncture in patients with low-level alpha-fetoprotein (AFP) liver cancer.

Methods: A total of 300 patients with primary liver cancer (PLC) (with AFP level $\leq 200 \mathrm{ng} / \mathrm{mL}$ and who underwent fine-needle aspiration) who were admitted to Central South University Xiangya School of Medicine Affiliated Haikou Hospital from January 2016 to December 2020 were selected to participate in the present study. Data, such as the expression of AFP and the biological characteristics of cells related to follow-up information, were retrospectively analyzed.

Results: Patients with AFP content $<50 \mathrm{ng} / \mathrm{mL}$ accounted for $27 \%$ of total patients. Patients with tumors $<20 \mathrm{~mm}$ accounted for $11 \%$ of total patients. There were 88 patients with $0-50 \mathrm{ng} / \mathrm{mL} \mathrm{AFP,} 75$ patients with 51-100 ng/mL AFP, 57 patients with 101-150 ng/mL AFP, and 83 patients with $200 \mathrm{ng} / \mathrm{mL}$ AFP. The sensitivity of detection was $\geq 90 \%$, specificity was $100 \%$, PPV was $100 \%$, and NPV was $\geq 90 \%$. In the present study, 34 patients with tumors $<20 \mathrm{~mm}$ in size underwent US-guided percutaneous transhepatic puncture. The sensitivity of the treatment was $93.33 \%, 100 \%$ specificity, $100 \% \mathrm{PPV}$, and $64.35 \% \mathrm{NPV}$. The sensitivity of US-guided percutaneous transhepatic puncture was $97.65 \%, 100 \%$ specificity, 100\% PPV, and $55.42 \% \mathrm{NPV}$ in 266 patients with tumor size $>20 \mathrm{~mm}$. Implantation and metastasis accounted for 5\% of complications, and gastrointestinal bleeding accounted for 7\%. Among the adverse reactions, nausea and vomiting accounted for $15 \%$, diarrhea accounted for $10 \%$, and bone marrow suppression accounted for $8 \%$.

Conclusions: US-guided percutaneous transhepatic puncture has high sensitivity, high specificity, and is relatively safe, with a low complication rate in patients with low-level AFP liver cancer, and has certain clinical diagnostic value.

Keywords: Liver cancer; alpha-fetoprotein (AFP); fine-needle aspiration; effectiveness; complications

Submitted Apr 08, 2021. Accepted for publication May 28, 2021.

doi: $10.21037 /$ tcr-21-701

View this article at: https://dx.doi.org/10.21037/tcr-21-701 


\section{Introduction}

Primary liver cancer (PLC) is a common malignant neoplasm worldwide. The incidence of PLC continues to increase due to the growing burden of global liver diseases (1). Because of its asymptomatic nature, liver cancer is usually diagnosed at late and advanced stages, at which time it is generally too late for surgical resection owing to tumor size, location of the tumor, or poor liver function, resulting in a short life expectancy (2). Therefore, disease monitoring and accurate diagnostic methods are important for the early diagnosis and treatment of high-risk patients.

Alpha-fetoprotein (AFP) is a serum glycoprotein produced by the embryonic yolk sac, and the fetal liver and is the most widely used biomarker to detect hepatocellular carcinoma (HCC) worldwide (3). It is also often used as an independent factor in predicting the overall survival of patients because of its high sensitivity, high specificity, and high accuracy in the patient's body. Pregnant women have higher blood AFP levels (4), whereas elevated serum AFP levels may be associated with a higher risk of tumors of gonadal origin, such as liver-like adenocarcinoma and HCC, and non-malignant chronic liver disease, including acute or chronic viral hepatitis $(5,6)$. Of HCC patients, $40 \%$ had normal AFP levels at initial diagnosis. AFP serum levels were reduced in $30 \%$ patients, despite AFP specificity. Therefore, clinical examinations, such as ultrasound (US)guided percutaneous transhepatic and radiological imaging, should be combined with AFP levels for HCC diagnosis.

US-guided puncture is a minimally invasive method for the diagnosis of HCC. Cytological and histological samples can be obtained by US or computed tomography (CT) guidance (7). Despite recent advances in imaging technology, the diagnostic value of US-guided percutaneous transhepatic puncture is still applied to optimal sensitivity and specificity, as well as future guidance, determination of chemotherapy regimens, and other treatments. A falsepositive diagnosis may still exist, even if AFP of $200 \mathrm{ng} / \mathrm{mL}$ is used as the diagnostic criterion, based on American Association for the Study of Liver Diseases guidelines, which may lead to unnecessary treatment and pain. A falsenegative diagnosis caused by a false explanation of low serum AFP cannot be ignored. Therefore, in the present study, we aimed to reassess the importance and determined the overall accuracy of serum AFP $\leq 200 \mathrm{ng} / \mathrm{mL}$ in a patient population as a diagnostic value in future clinical practice by percutaneous transhepatic puncture guided by US. We present the following article in accordance with the STARD reporting checklist (available at https://dx.doi.org/10.21037/ tcr-21-701).

\section{Methods}

\section{General information}

A total of 300 patients with PLC, who were treated in Central South University Xiangya School of Medicine Affiliated Haikou Hospital from January 2016 to December 2020, were analyzed retrospectively. Inclusion criteria were as follows: (I) PLC diagnosed by CT, magnetic resonance imaging (MRI), and histopathological biopsy; (II) AFP content $\leq 200 \mathrm{ng} / \mathrm{mL}$; and (III) patients who had undergone US-guided percutaneous transhepatic puncture. Exclusion criteria were as follows: (I) pregnant or lactating; (II) mental illness; (III) coagulation dysfunction; (IV) serious liver and kidney function disorders; and (V) lost to follow up. All procedures performed in this study involving human participants were in accordance with the Declaration of Helsinki (as revised in 2013). The study was approved by the Ethics Committee of Central South University Xiangya School of Medicine Affiliated Haikou Hospital (No.: 2014HN096) and informed consent was taken from all the patients.

\section{Observation indicators}

Observation indicators were as follows: (I) comparison of patients' general clinical data; (II) analysis of diagnostic results in patients with liver cancer with different AFP expression levels; and (III) effect of US-guided percutaneous transhepatic puncture on diagnostic results for different tumor sizes.

\section{Statistical analysis}

Data were statistically analyzed by GraphPad Prism 8.0 (GraphPad Software Inc., San Diego, CA, USA) to calculate sensitivity, specificity, positive predictive value (PPV), negative predictive value (NPV), and overall diagnostic accuracy (compared with the final diagnosis after follow up). Measurement data were represented as means \pm standard deviations and analyzed by $t$-test. Counting data were expressed as percentages and analyzed by $\chi^{2}$-test. $\mathrm{P}<0.05$ was considered statistically significant. 
Table 1 Comparison of patients' general clinical data

\begin{tabular}{|c|c|c|}
\hline Variable & Cases (n) & $\%$ \\
\hline \multicolumn{3}{|l|}{ Sex } \\
\hline Male & 205 & 68 \\
\hline Female & 95 & 32 \\
\hline \multicolumn{3}{|c|}{$\begin{array}{l}\text { Alpha-fetoprotein expression } \\
\text { level }(\mathrm{ng} / \mathrm{mL})\end{array}$} \\
\hline$<50$ & 80 & 27 \\
\hline $50-100$ & 75 & 25 \\
\hline $100-150$ & 73 & 24 \\
\hline 150-200 & 72 & 24 \\
\hline \multicolumn{3}{|c|}{ Tumor size (mm) } \\
\hline$<20$ & 34 & 11 \\
\hline $20-50$ & 27 & 9 \\
\hline 50-80 & 51 & 17 \\
\hline $80-100$ & 99 & 33 \\
\hline$>100$ & 89 & 30 \\
\hline \multicolumn{3}{|l|}{ Cirrhosis } \\
\hline Yes & 169 & 56 \\
\hline No & 131 & 44 \\
\hline
\end{tabular}

\section{Results}

\section{Comparison of patients' general clinical data}

The data analysis showed that $32 \%$ of the patients with AFP liver cancer were women. Different AFP expression levels were also found among patients. Patients with AFP levels $50 \mathrm{ng} / \mathrm{mL}$ accounted for less than $50 \%$ of all patients, those with AFP levels $50-100 \mathrm{ng} / \mathrm{mL}$ accounted for $25 \%$ of all patients, those with AFP levels $100-150 \mathrm{ng} / \mathrm{mL}$ accounted for $25 \%$ of all patients, and those with AFP levels 150-200 accounted for $24 \%$ of all patients. Cancer patients with tumors $<20 \mathrm{~mm}$ accounted for $11 \%$ of total patients, those with tumors $20-50 \mathrm{~mm}$ accounted for $9 \%$ of the total, those with tumors $50-80 \mathrm{~mm}$ accounted for $17 \%$ of the total, those with tumors $80-100 \mathrm{~mm}$ accounted for $33 \%$ of the total, and those with tumors $>100 \mathrm{~mm}$ accounted for $30 \%$ of the total. In total, $56 \%$ of patients had cirrhosis. The statistical analysis data are shown in Table 1.

\section{Diagnostic analysis results of HCC patients with different AFP expression levels}

A total of 88 patients had an AFP level of $0-50 \mathrm{ng} / \mathrm{mL}$, 75 patients had an AFP level of 51-100 ng/mL, 57 had an AFP level of $101-150 \mathrm{ng} / \mathrm{mL}$, and 83 patients had an AFP of $151-200 \mathrm{ng} / \mathrm{mL}$. The sensitivity of detection was $\geq 90 \%$, specificity was $100 \%$, PPV was $100 \%$, and NPV was $\geq 90 \%$ (Table 2).

\section{Effect of US-guided percutaneous transhepatic puncture on} diagnostic results for different tumor sizes

Thirty-four patients had a tumor size $<20 \mathrm{~mm}$. The sensitivity of US-guided percutaneous transhepatic puncture was $93.33 \%, 100 \%$ specificity, $100 \% \mathrm{PPV}$, and $64.35 \%$ NPV. A total of 266 patients had a tumor size $>20 \mathrm{~mm}$. The sensitivity of US-guided percutaneous transhepatic puncture was $97.65 \%, 100 \%$ specificity, $100 \% \mathrm{PPV}$, and $55.42 \%$ NPV (Table 3).

\section{Analysis of complications and adverse reactions caused by US-guided percutaneous transhepatic puncture}

US-guided percutaneous transhepatic diagnosis accounted for $5 \%$ of complications in patients with AFP liver cancer and $7 \%$ of gastrointestinal bleeding. Nausea and vomiting accounted for $15 \%$, diarrhea $10 \%$, and bone marrow suppression $8 \%$ (Table 4).

\section{Discussion}

The accuracy of cancer diagnosis is important, as the treatment will vary based on the type of cancer. The incorrect diagnosis may result in a wrong or delayed treatment harm to the patient. Over the past several decades, there has been controversy concerning the role of US-guided percutaneous transhepatic puncture in the detection of HCC. Advances in dynamic imaging techniques have improved the accuracy of HCC diagnosis in most nodules, and most of these dynamic imaging techniques are based on angiogenesis and metastasis (8). CT and MRI have high sensitivity (55-91\%) and specificity in diagnosing HCC (77-96\%) (9). However, there are some limitations in assessing HCC occurrence when only 
Table 2 Diagnostic results of liver cancer patients with different expression levels of alpha-fetoprotein

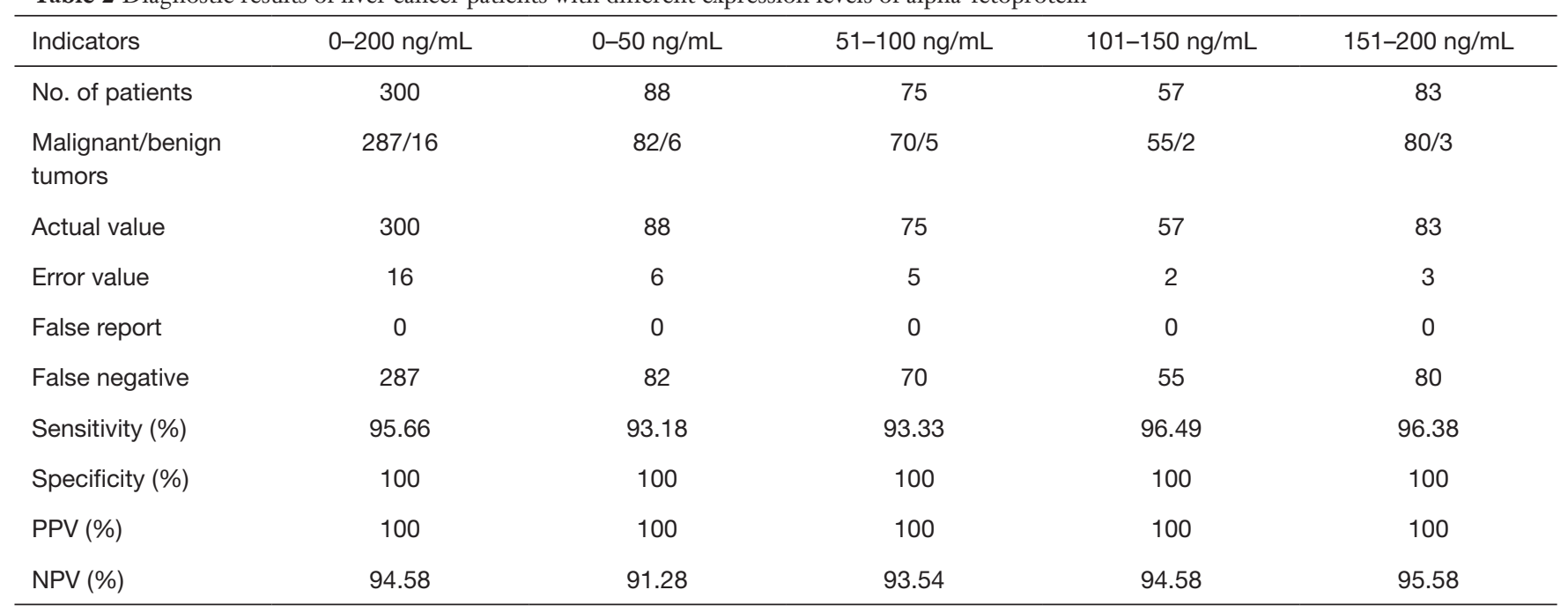

NPV, negative predictive value; PPV, positive predictive value.

Table 3 Impact of ultrasound-guided percutaneous transhepatic puncture on the diagnostic results of different tumor sizes

\begin{tabular}{lcc}
\hline Indicators & $<20 \mathrm{~mm}$ & $>20 \mathrm{~mm}$ \\
\hline No. cases & 34 & 266 \\
Malignant/benign tumors & $30 / 4$ & $256 / 10$ \\
Sensitivity (\%) & 93.33 & 97.65 \\
Specificity (\%) & 100 & 100 \\
PPV (\%) & 100 & 100 \\
NPV (\%) & 64.35 & 55.42 \\
\hline
\end{tabular}

NPV, negative predictive value; PPV, positive predictive value.

Table 4 Complications and adverse reactions caused by ultrasoundguided percutaneous transhepatic puncture diagnosis

\begin{tabular}{lcc}
\hline Clinical symptoms & Cases (n) & $\%$ \\
\hline Implantation transfer & 15 & 5 \\
Gastrointestinal bleeding & 20 & 7 \\
Nausea and vomiting & 45 & 15 \\
Diarrhea & 30 & 10 \\
Bone marrow suppression & 25 & 8 \\
\hline
\end{tabular}

using angiogenesis and metastasis. For example, enhanced patterns of small HCC depend on tumor sizes and cell differentiation, and tumors $<2 \mathrm{~cm}$ may exhibit atypical enhancement. Subvascular tumors are likely to be ignored in that HCC diagnosis is based on vascular type; $52 \%$ of early arteriole enhancement lesions decrease over time and are considered false lesions (10). In the present study, 34 patients with tumors $<20 \mathrm{~mm}$ in size underwent US-guided percutaneous transhepatic puncture. The sensitivity of the treatment was $93.33 \%, 100 \%$ specificity, $100 \% \mathrm{PPV}$, and $64.35 \% \mathrm{NPV}$. The sensitivity of US-guided percutaneous transhepatic puncture was $97.65 \%, 100 \%$ specificity, $100 \%$ PPV, and $55.42 \% \mathrm{NPV}$ in 266 patients with tumor size $>20 \mathrm{~mm}$. There was no significant difference between tumors $<2 \mathrm{~mm}$ and those $\geq 2 \mathrm{~mm}$ in the subgroups based on sensitivity, specificity, PPV, and NPV, which indicates the advantage of US-guided percutaneous transhepatic puncture for the diagnosis of small hepatic lesions.

US-guided percutaneous transhepatic puncture is the most commonly used confirmatory diagnostic method for HCC (11). Although this is a minimally invasive diagnostic approach, it still has serious side-effects and may aggravate patients' pain (12). Because AFP and USguided percutaneous transhepatic puncture are common diagnostic methods for a confirmed diagnosis of PLC, it is important to assess the effectiveness and relevance of these two diagnostic methods to avoid unnecessary surgery and patient pain. Unfortunately, the value of US-guided percutaneous liver puncture in patients with serum AFP $\leq 200 \mathrm{ng} / \mathrm{mL}$ is rarely studied (13). The results of the present study indicated that 88 patients had $0-50 \mathrm{ng} / \mathrm{mL}$ AFP levels, 75 had 51-100 ng/mL AFP levels, 57 had 101$150 \mathrm{ng} / \mathrm{mL}$ AFP levels, and 83 had 151-200 ng/mL AFP 
levels. Detection of sensitivity was $90 \%, 100 \%$ specificity, $100 \% \mathrm{PPV}$, and $\geq 90 \% \mathrm{NPV}$, indicating that changes in AFP content do not affect the accuracy of US-guided percutaneous transhepatic diagnosis.

Pathological diagnosis can provide a variety of benefits for patients looking for appropriate treatment options for suspicious malignant tumors. US-guided percutaneous transhepatic puncture can provide pathological samples for early diagnosis and data for medical research. In addition, for patients with suspected small liver cancer, especially with atypical imaging lesions, once liver nodules are found in patients, US-guided percutaneous transhepatic puncture can alleviate patients' anxiety. Early detection of liver nodules can decrease the cost of long-term imaging surveillance and enable early treatment options.

In previous report (14), biliary peritonitis was found to be a serious complication of US-guided transhepatic puncture. It is noteworthy that bile peritonitis was not detected in any patient in the present study, which could possibly be due to the inevitable avoidance of aspiration in cases of bile duct punctures. According to the data reported from recent study, US-guided percutaneous transhepatic diagnosis accounted for $5 \%$ of complications in patients with AFP liver cancer, $7 \%$ of gastrointestinal bleeding, $15 \%$ of nausea and vomiting, $10 \%$ of diarrhea, and $8 \%$ of bone marrow suppressions. Moreover, bleeding is usually associated with severe cirrhosis or large superficial tumors not covered by normal hepatic parenchyma, rather than coagulation curves and platelet counts (15). When the tumor is covered with sufficient liver parenchyma, it can still be punctured, regardless of tumor size, and there is no excessive risk of bleeding (16). For tumors that have relatively large or small coverage of normal liver tissue, bleeding after percutaneous liver puncture under US guidance should be carefully monitored. The risk of bleeding in each patient should be observed before therapy. If the risk of bleeding is high, imaging should be undertaken for diagnosis to avoid bleeding after US-guided percutaneous liver puncture.

In conclusion, US-guided percutaneous transhepatic puncture has a good clinical diagnostic value in patients with low-level AFP liver cancer because of its high sensitivity, high specificity, high accuracy, and relatively safe and low incidence of complications.

\section{Acknowledgments}

Funding: None.

\section{Footnote}

Reporting Checklist: The authors have completed the STARD reporting checklist. Available at https://dx.doi. org/10.21037/tcr-21-701

Data Sharing Statement: Available at https://dx.doi. org/10.21037/tcr-21-701

Conflicts of Interest: All authors have completed the ICMJE uniform disclosure form (available at https://dx.doi. org/10.21037/tcr-21-701). The authors have no conflicts of interest to declare.

Ethical Statement: The authors are accountable for all aspects of the work in ensuring that questions related to the accuracy or integrity of any part of the work are appropriately investigated and resolved. All procedures performed in this study involving human participants were in accordance with the Declaration of Helsinki (as revised in 2013). The study was approved by the Ethics Committee of Central South University Xiangya School of Medicine Affiliated Haikou Hospital (No.: 2014HN096) and informed consent was taken from all the patients.

Open Access Statement: This is an Open Access article distributed in accordance with the Creative Commons Attribution-NonCommercial-NoDerivs 4.0 International License (CC BY-NC-ND 4.0), which permits the noncommercial replication and distribution of the article with the strict proviso that no changes or edits are made and the original work is properly cited (including links to both the formal publication through the relevant DOI and the license). See: https://creativecommons.org/licenses/by-nc-nd/4.0/.

\section{References}

1. Wang XX, Qi QG. Research Progress in the Treatment of Primary Liver Cancer. World Latest Med Information 2019;19:52-4.

2. Sun Y, Zhang Z. In Silico Identification of Crucial Genes and Specific Pathways in Hepatocellular Cancer. Genet Test Mol Biomarkers 2020;24:296-308.

3. Luo P, Wu S, Yu Y, et al. Current Status and Perspective Biomarkers in AFP Negative HCC: Towards Screening for and Diagnosing Hepatocellular Carcinoma at an Earlier Stage. Pathol Oncol Res 2020;26:599-603. 
4. Yue CY, Zhang B, Ying CM. Elevated Serum Level of IL35 Associated with the Maintenance of Maternal-Fetal Immune Tolerance in Normal Pregnancy. PLoS One 2015;10:e0128219.

5. Zou M, Li Y, Dai Y, et al. AFP-producing hepatoid adenocarcinoma (HAC) of peritoneum and omentum: a case report and literature review. Onco Targets Ther 2019;12:7649-54.

6. Muroyama Y, Tamiya H, Tanaka G, et al. AlphaFetoprotein-Producing Lung Hepatoid Adenocarcinoma with Brain Metastasis Treated with S-1. Case Rep Oncol 2020;13:1552-9.

7. Li X, Yu J, Liang P, et al. Ultrasound-guided percutaneous microwave ablation assisted by three-dimensional visualization operative treatment planning system and percutaneous transhepatic cholangial drainage with intraductal chilled saline perfusion for larger hepatic hilum hepatocellular $(\mathrm{D} \geq 3 \mathrm{~cm})$ : preliminary results. Oncotarget 2017;8:79742-9.

8. Ippolito D, Pecorelli A, Querques G, et al. Dynamic Computed Tomography Perfusion Imaging: Complementary Diagnostic Tool in Hepatocellular Carcinoma Assessment From Diagnosis to Treatment Follow-up. Acad Radiol 2019;26:1675-85.

9. Wang G, Zhu S, Li X. Comparison of values of CT and MRI imaging in the diagnosis of hepatocellular carcinoma and analysis of prognostic factors. Oncol Lett 2019;17:1184-8.

10. Shimizu A, Koike S, Ito K, et al. Cirrhosis or Chronic Hepatitis: Evaluation of Small ( $\leq 2-\mathrm{cm})$ Early-Enhancing Hepatic Lesions with Serial Contrast-enhanced Dynamic

Cite this article as: Fu Y, Chen J, Li C, Chen L, Zhang Z, Huang Z. Effectiveness of ultrasound-guided percutaneous transhepatic puncture for the diagnosis of low-level alphafetoprotein liver cancer patients. Transl Cancer Res 2021;10(6):2985-2990. doi: 10.21037/tcr-21-701
MR Imaging1. Radiology 2003;226:550-5.

11. Schmitz D, Weller N, Doll M, et al. An Improved Method of Percutaneous Transhepatic Biliary Drainage Combining Ultrasound-Guided Bile Duct Puncture with Metal Stent Implantation by Fluoroscopic Guidance and Endoscopic Visualization as a One-Step Procedure: A Retrospective Cohort Study. J Clin Int Radiol Isvir 2018;2:135-43.

12. Zhao QM, Huang MF, Ming LU, et al. Value of ultrasound-guided percutaneous transhepatic gallbladder puncture in the treatment of elderly patients with acute cholecystitis. Hainan Medical Journal 2019;30:1584-96.

13. Chen QW, Cheng CS, Chen H, et al. Effectiveness and complications of ultrasound guided fine needle aspiration for primary liver cancer in a Chinese population with serum $\alpha$-fetoprotein levels $\leq 200 \mathrm{ng} / \mathrm{ml}$--a study based on 4,312 patients. PLoS One 2014;9:e101536.

14. Giurazza F, Corvino F, Contegiacomo A, et al. Safety and effectiveness of ultrasound-guided percutaneous transhepatic biliary drainage: a multicenter experience. J Ultrasound 2019;22:437-45.

15. Gado A, Ebeid B, Axon A, et al. Prevalence and outcome of peptic ulcer bleeding in patients with liver cirrhosis. Alexandria J Med 2014;50:143-8.

16. Petit A, Hocquelet A, N'kontchou G, et al. No-Touch Multi-bipolar Radiofrequency Ablation for the Treatment of Subcapsular Hepatocellular Carcinoma $\leq 5 \mathrm{~cm}$ Not Puncturable via the Non-tumorous Liver Parenchyma. Cardiovasc Intervent Radiol 2020;43:273-83.

(English Language Editor: R. Scott) 